\title{
Advanced Signal Processing for Wireless Multimedia Communications
}

\author{
Xiaodong Wang \\ Texas A \& M University
}

\begin{abstract}
There is at present a worldwide effort to develop next-generation wireless communication systems. It is envisioned that many of the future wireless systems will incorporate considerable signal-processing intelligence in order to provide advanced services such as multimedia transmission. In general, wireless channels can be very hostile media through which to communicate, due to substantial physical impediments, primarily radiofrequency interference and time-arying nature of the channel. The need of providing universal wireless access at high data-rate (which is the aim of many merging wireless applications) presents a major technical challenge, and meeting this challenge necessitates the development of advanced signal processing techniques for multiple-access communications in non-stationary interference-rich environments.

In this paper, we present some key advanced signal processing methodologies that have been developed in recent years for interference suppression in wireless networks. We will focus primarily on the problem of jointly suppressing multiple-access interference (MAI) and intersymbol interference (ISI), which are the limiting sources of interference for the high data-rate wireless systems being proposed for many emerging application areas, such as wireless multimedia. We first present a signal subspace approach to blind joint suppression of MAI and ISI. We then discuss a powerful iterative technique for joint interference suppression and decoding, so-called Turbo multiuser detection, that is especially useful for wireless multimedia packet communications. We also discuss space-time processing methods that employ multiple antennas for interference rejection and signal enhancement. Finally, we touch briefly on the problems of suppressing narrowband interference and impulsive ambient noise, two other sources of radio-frequency interference present in wireless multimedia networks.
\end{abstract}

Keywords: wireless communications, interference rejection, signal processing

\section{Introduction}

\section{Potential Applications of Future Wireless Mobile System}

The coming generation of wireless communication technology promises a giant leap forward in formation accessibility. Advanced features of wireless mobile systems, including data rates compatible with multimedia applications, global roaming capability, and coordination with other network structures, will enable many emerging applications such as:

Material published as part of this journal, either on-line or in print, is copyrighted by the publisher of Informing Science. Permission to make digital or paper copy of part or all of these works for personal or classroom use is granted without fee provided that the copies are not made or distributed for profit or commercial advantage AND that copies 1) bear this notice in full and 2) give the full citation on the first page. It is permissible to abstract these works so long as credit is given. To copy in all other cases or to republish or to post on a server or to redistribute to lists requires specific permission and payment of a fee. Contact Editor@inform.nu to request redistribution permission.
- Virtual navigation: The graphical representation of the physical characteristics of an environment, such as streets, buildings, etc., is stored in a remote database. Blocks of this database are transmitted rapidly to a vehicle, where a program renders them so the occupants can visualize the environment ahead.

- Tele-medicine: The paramedic assisting the patient in a remote location must communicate the medical records with the hospital and may need videoconference assistance from a doctor for instructions.

- Crisis management: When severe nature disasters occur and the entire communications infrastructure is in disarray, restoring communications quickly is vital. With wideband wireless mobile ad hoc network, limited and even total communications capability, including internet and video services, could be set up in hours instead of days or even weeks required for restoration of wireline communications. 
- Distance learning: Wideband wireless communications will provide cost-effective educational opportunities available on the Internet, for people living in thinly populated or remote areas.

\section{The Role of Signal Processing for Wireless Multimedia Communications}

It is anticipated that the future-generation wireless communication systems will incorporate considerable signal-processing intelligence in order to provide advanced services such as multimedia. In order to make optimal use of available bandwidth for these services and to provide maximal flexibility, many such systems will operate as multiple-access systems, in which channel bandwidth is shared by many users on a randomaccess basis using protocols such as code-division multipleaccess (CDMA) signaling. Moreover, in order to support the high data rates inherent in such services, ratios of bit rates to bandwidths will be pushed to their limits. In general, wireless channels can be very hostile media through which to communicate. Physical impairments such as multiple-access interference, co-channel interference, multipath transmission, amplitude fading and dispersion due to limited bandwidth, all contribute to make it difficult to transmit data reliably and quickly through wireless channels. Moreover, the dynamism resulting from user mobility and the above-noted random-access nature of mobile channels, amplify the effects of these impairments, and make them much more difficult to ameliorate. Solution to these difficulties lie in the use of advanced signal processing techniques, and in this paper we present some of the recently developed methodologies for interference rejection that are especially useful for wireless multimedia communications.

\section{Outline of this Article}

In particular, we will focus on interference rejection techniques for code-division multiple-access (CDMA) wireless systems. CDMA is the multiple-access scheme adopted in the IS-95 digital cellular standard, and it is the preferred mode of multiaccessing in many current and emerging wireless applications, such as third-generation mobile telephony and wireless personal communications. Our discussion will be primarily focused on the problem of jointly suppressing multiple-access interference (MAI) and intersymbol interference (ISI), which are the limiting sources of interference for high data-rate wireless applications, such as wireless multimedia. After describing the general problem of interference rejection in wireless communications, we present a signal subspace approach to blind joint suppression of MAI and ISI. We then discuss a powerful iterative technique for joint interference suppression and decoding, so-called Turbo multiuser detection, that is especially useful for wireless multimedia packet communications. We also discuss space-time processing methods that employ multiple antennas for interference rejection and signal enhancement.

Finally, we touch briefly on the problems of suppressing narrowband interference and impulsive ambient noise, two other sources of radio-frequency interference present in wireless multimedia networks.

\section{The Problem of Interference Rejection in Wireless Communications}

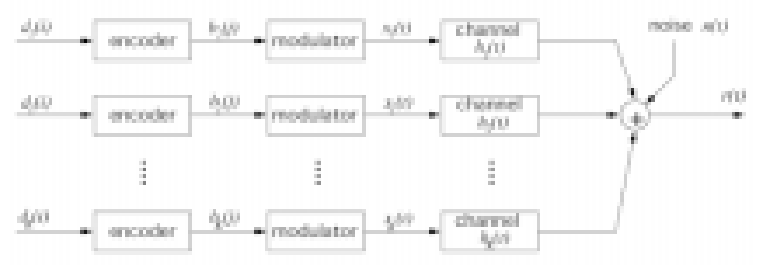

Figure 1: Systematic illustration of a multiple-access wireless digital communications system.

Figure 1 is the systematic illustration of a multiple-access digital communication system typically employed in modern wireless networks. In this system, there are $K$ users transmitting information-bearing signals through a wireless medium. Each user's digital data (e.g., $\left\{d_{k}(i)\right\}$ which may represent voice, image, video or multimedia information, are first encoded using some error-correct coding schemes to protect them from being corrupted by the channel during transmission. The coded data of each user (e.g., $\left.\left\{b_{k}(j)\right\}\right)$ are then mapped by a modulator onto signals that can be efficiently transmitted over the communication channel. Each modulated signal (e.g., $\left.x_{k}(t)\right)$ is then transmitted through a wireless channel, whose characteristics can be mathematically represented by a function $h_{k}(t)$. At the receiver end, the received signal is the superposition of the $\$ K \$$ users' channel-distorted transmitted signals, plus the ambient channel noise \$n(t)\$. Mathematically, the received signal can be written as:

$$
\begin{aligned}
& r(t)=\sum_{k=1}^{K} \int_{-\infty}^{\infty} h_{k}(t-u) x_{k}(u) \mathrm{d} u+n(t) \\
& =\sum \int_{-\infty}^{\infty} h_{k}(t-u) f_{k}\left(u ;\left\{d_{k}(i), \text { enc }_{k}, \bmod _{k}\right) \mathrm{d} u+n(t)\right.
\end{aligned}
$$

where we write the $k$-th user's transmitted signal $\mathrm{x}_{\mathrm{k}}(u)$ as $f_{k}(u$; $\left\{d_{\mathrm{k}}(i)\right\},\left\{\mathbf{e n c}_{\mathrm{k}}, \boldsymbol{m o d}_{k}\right)$ to highlight that it is a function of the information data, the channel encoder and the modulator of that user.

Our problem at hand is to reproduce the information data of one or some or all users based on the received signal $r(t)$. For 
example, in a cellular telephony system, $r(t)$ may represent the signal received by a particular mobile phone. Let us call the owner of that phone User 1 . Then $\left\{d_{l}(i)\right\}$ represents the digital voice information data for that user. In order to recover $\left\{d_{l}(i)\right\}$ from the receive signal, the only prior information available at User 1's receiver is the structures of the encoder and modulator at the corresponding transmitter, i.e., $\left(\mathbf{e n c}_{1}, \bmod _{1}\right)$, but nothing else. As another example, $r(t)$ may also represent the signal received by a basestation receiver in a wireless cellular network. In this case, all users' information data $\left\{d_{k}(i), k=1, \ldots, K\right\}$ must be recovered. The receiver has the knowledge of the structures of the encoder and modulator of all users, $\left\{\left(\mathbf{e n c}_{\mathrm{k}}\right.\right.$, $\left.\left.\bmod _{\mathrm{k}}\right), k=1, \ldots, K\right\}$, but not that of the characteristics of the channels, i.e., $\left\{h_{k}(t), k=1, \ldots K\right\}$.

This problem of signal recovery in a multiple-access system is of great importance both in theory and in practice. The current engineering solution is to make the user signals well separated either in time or in frequency such that effectively $K=1$ in our model. The obvious drawback of this approach is the substantial loss of spectral efficiency, i.e., the number of users that can be supported in a network is substantially reduced. With the explosive growth in demand for advanced wireless services, significant amount of recent research in both universities and industry has addressed this problem. In what follows we present some of the recently developed advanced signal processing techniques that address various aspects of the problem of interference rejection in wireless communications.

\section{Subspace Blind and Group-Blind Multi- user Detection}

Our problem at hand is to recover one or some of the users' transmitted symbols $\left\{b_{k}(i)\right\}$ based on the received signal $r(t)$ [see Figure 1]. By appropriately filtering and sampling the received waveform $r(t)$ at the receiver front-end, we convert the continuous-time signal $r(t)$ into a discrete-time signal $\{\boldsymbol{r}(i)$ \}. It can be shown that [24,27] mathematically $\boldsymbol{r}(i)$ can be represented as

$$
\mathbf{r}(i)=\mathbf{H b}(i)+\mathbf{n}(i)
$$

where $\mathbf{H}$ is a matrix which is determined by the transmitted modulation waveforms and the physical channel characteristics of all users; $\$\{\backslash b f b\}(i) \$$ is a vector which contains the transmitted symbols of all users at times $(i-1), i$ and $(i+1)$, i.e., $\left\{b_{k}(j): j=i-1, i, i+1, k=1, \ldots K\right\}$; and $\mathbf{n}(i)$ is a vector of channel ambient noise samples. Note that the received signal $\mathbf{r}(i)$ contains not only the desired user's signal, but also signal from other users -- the so-called multiple-access interference (MAI). Moreover, $\mathbf{r}(i)$ contains not only the symbols at time $i$, but also symbols from time $(i-1)$ and $(i+1)$-- the so-called $i n-$ tersymbol interference (ISI).

A linear detector for the $k$-th user can be represented by a vector $\mathbf{w}_{\mathrm{k}}$ which is applied to the received signal $\mathbf{r}(i)$ in (2), to compute the $i$-th bit of the $k$-th user, according to the following rule:

$$
\hat{b}_{k}[i]=\operatorname{sgn}\left\{\mathfrak{R}\left(\mathrm{w}_{k}^{\mathrm{H}} \mathrm{r}[i]\right)\right\}
$$

One type of such a linear detector is the so-called linear MMSE detector, where w $k$ is chosen to minimize the output mean-square error (MSE), i.e.,

$$
\mathrm{W} k=\arg \min _{\mathrm{w}} E\left\{\left|b_{k}[i]-\mathrm{w}^{H} \mathrm{r}[i]\right|^{2}\right\}
$$

The problem of finding the linear detector for the $k$-th user is difficult because the channel matrix $\mathbf{H}$ is unknown to the receiver. Recall that the receiver only knows one or some users' modulation waveforms (cf. Figure 1). If only the $k$-user's modulation waveform is known, then the problem of computing $\mathbf{w}_{\mathrm{k}}$ is referred to as the problem of blind multiuser detection. On the other hand, if the $k$-th user's modulation waveform, together with those of some other users (but not all users), is known, then the problem is referred to as the problem of group-blind multiuser detection.

The problems of blind multiuser detection and group-blind multiuser detection have been recently solved in [24,25] and [10] respectively. The basic idea is to identify the so-called signal subspace spanned by the multiuser signals using some adaptive subspace tracking algorithms. Based on such an estimated signal subspace, the physical channel responses of the desired users can be then estimated, and the corresponding blind or group-blind multiuser detectors for those users can also be obtained.

\section{Simulation Example}

We next provide computer simulation results to demonstrate the performance of the blind and group-blind linear multiuser detectors in a wireless CDMA networks. The simulated system is an asynchronous CDMA system with processing gain 15. The $m$-sequences of length 15 and their shifted versions are employed as the user spreading sequences. The chip pulse is a raised cosine pulse with roll-off factor 0.5 . The initial delay of each user is uniform on $\left[0,4 T_{\mathrm{c}}\right]$, where $T_{\mathrm{c}}$ is the chip interval. Each user's channel has three paths. The delay of each path is uniform on $\left[0,6 T_{\mathrm{c}}\right]$. The fading gain of each path in each user's channel is generated from a complex Gaussian distribution and fixed for all simulations. The path gains in each user's channel 


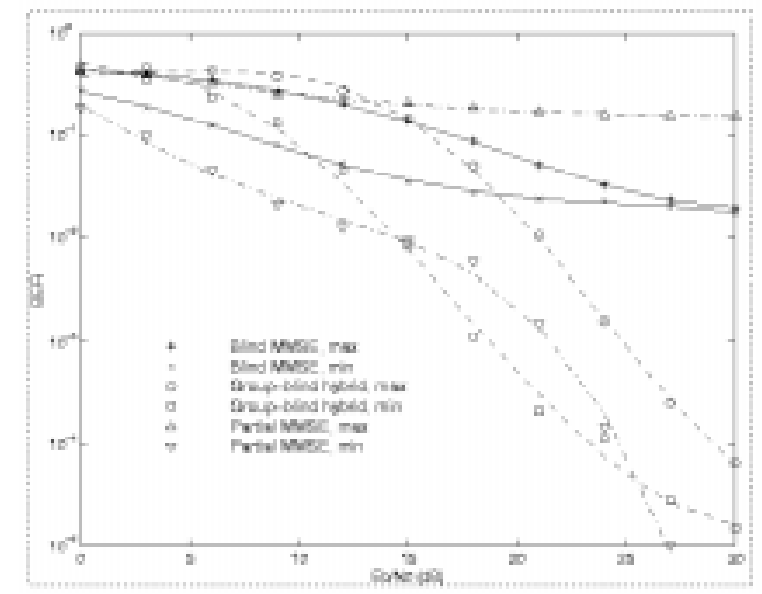

Figure 2: Comparison of the performance of the blind and the group-blind linear detectors.

are normalized so that each user's signal arrives at the receiver with the same power. The number of users in the simulation is 10, with 7 known users.

In Figure 2, we plot the bit error rate (BER) curves for the blind linear MMSE detector, the group-blind linear hybrid detector, and a partial MMSE detector.

The partial MMSE detector ignores the unknown users and forms the linear MMSE detector for the known users only. For each of these detectors, and for each value of signal-to-noise ratio $\left(E_{\mathrm{b}} / N_{\mathrm{o}}\right)$, the minimum and the maximum BER among the 7 known users is plotted. It is seen that the group-blind detector significantly outperforms the blind MMSE detector and the partial MMSE detector. Indeed the blind MMSE detector exhibits an error floor at high $\left(E_{\mathrm{b}} / N_{\mathrm{o}}\right)$ values. This is due to the finite length of received signal frame, based on which the detector is estimated. The group-blind hybrid MMSE detectors do not show error floors in the BER range considered here. Of course, due to the finite signal frame length, the group-blind detectors also have error floors. But such floors are much lower that that of the blind linear MMSE detector

\section{Turbo Multiuser Detection}

Most communication systems employ error correcting coding to prevent the transmitted data from being corrupted by the channel. This is especially true for wireless voice/data/multimedia communications, where the physical channels are particularly severe. Coding introduces additional signal structures that can be exploited by interference rejection algorithms. In what follows we present an iterative technique for joint interference suppression and decoding, so-called Turbo multiuser detection, that is especially useful for wireless packet multimedia communications. Recently iterative ("Turbo") processing techniques have received considerable attention followed by the discovery of the powerful Turbo codes $[4,5]$. The so called Turbo-principle can be successfully applied to many detection/decoding problems such as serial concatenated decoding, equalization, coded modulation, multiuser detection and joint source and channel decoding [9].

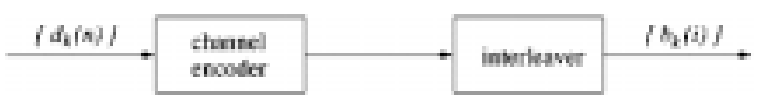

Figure 3: The channel encoder and interleaver of the $k$-th user.

Consider again the multiple-access system depicted in Figure 1. Let's assume that each user's transmitted binary symbols $\left\{b_{k}(i)\right\}$ are the outputs of a channel encoder followed by a interleaver, as shown in Figure 3. The input to the channel encoder are the information bits of that user $\left\{d_{k}(n)\right\}$. The purpose of the interleaver is to reduce the influence of the error bursts caused by the channel. A Turbo multiuser receiver has been received developed in [28]. This receiver structure is shown in Figure 4. It consists of two stages: a soft-input soft output (SISO) multiuser detector, followed by $K$ parallel single-user SISO channel decoders. The two stages are separated by deinterleavers and interleavers. The SISO multiuser detector delivers the a posteriori log-likelihood ratio (LLR) of a transmitted " +1 " and a transmitted " -1 " for every code bit of every user,

$$
\Lambda_{1}\left[b_{k}(i)\right] \stackrel{\Delta}{=} \log \frac{P\left[b_{k}(i)=+1 \mid r(t)\right]}{P\left[b_{k}(i)=-1 \mid r(t)\right]} .
$$

Using Bayes' rule, (5) can be written as

$$
\Lambda_{1}\left[b_{k}(i)\right]=\underbrace{\frac{p\left[r(t) \mid b_{k}(i)=+1\right]}{p\left[r(t) \mid b_{k}(i)=-1\right]}}_{\lambda_{1}\left[b_{k}(i)\right]}+\log \frac{P\left[b_{k}(i)=+1\right]}{\underbrace{P\left[b_{k}(i)=-1\right]}_{\lambda_{2}^{P}\left[b_{k}(i)\right]}},
$$

where the second term in (6), denoted by $\lambda_{2}{ }^{\mathrm{P}}\left[b_{k}(i)\right]$, represents the a priori LLR of the code bit $b_{k}(i)$, which is computed by the channel decoder of the $k$-th user in the previous iteration, interleaved and then fed back to the SISO multiuser detector. (The superscript ${ }^{\mathrm{p}}$ indicates the quantity obtained from the previous iteration). For the first iteration, assuming equally likely code bits, i.e., no prior information available, we then have $\lambda_{2}{ }^{\mathrm{p}}\left[b_{k}(i)\right]$, for $1 \leq \mathrm{k} \leq \mathrm{K}$ and $0 \leq i<M$. The first term in (6), denoted by $\lambda_{1}\left[b_{k}(i)\right]$, represents the extrinsic information delivered by the SISO multiuser detector, based on the received signal $r(t)$, the structure of the multiuser signal given by (2), the prior information about the code bits of all other users, $\left\{\lambda_{2}^{p}\left[b_{l}(j)\right], l \neq k, 0 \leq j<M\right\}$, and the prior information about the code bits of the $k$-th user other than the $i$-th bit, $\left\{\lambda_{2}^{p}\left[b_{k}(j)\right]\right.$, $j \neq I\}$. The extrinsic information $\lambda_{1}\left[b_{k}(i)\right]$ which is not influenced by the a priori information $\left\{\lambda_{2}{ }^{p}\left[b_{k}(i)\right]\right.$ provided by the 
channel decoder, is then reverse interleaved and fed into the $k$ th user's channel decoder, as the a priori information in the next iteration.

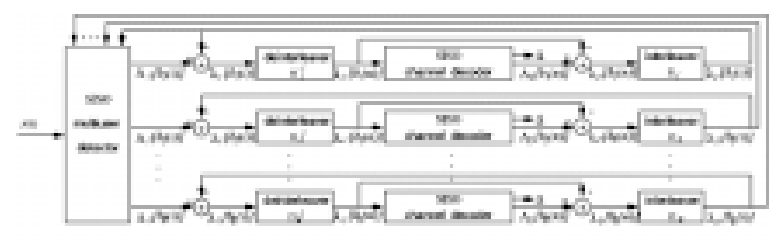

Figure 4: A Turbo multiuser receiver for coded CDMA.

Based on the prior information $\lambda_{1}^{p}\left[b_{k}(n)\right]$, and the trellis structure (i.e., code constraints) of the channel code, the $k$-th user's SISO channel decoder computes the a posteriori LLR of each code bit,

$$
\begin{aligned}
& \Lambda_{2}\left[b_{k}(n)\right] \stackrel{\Delta}{=} \log \frac{\left.P\left[b_{k}(n)=+1 \mid \lambda_{1}^{p}\left[b_{k}(n)\right]\right\}_{n=0}^{M-1} ; \text { decoding }\right]}{\left.P\left[b_{k}(n)=-1 \mid \lambda_{1}^{p}\left[b_{k}(n)\right]\right\}_{n=0}^{M-1} ; \text { decoding }\right]} \\
& =\lambda_{2}\left[b_{k}(n)\right]+\lambda_{1}^{p}\left[b_{k}(n)\right] .
\end{aligned}
$$

It is seen from (7) that the output of the SISO channel decoder is the sum of the prior information $\lambda_{1}{ }^{p}\left[b_{k}(n)\right]$, and the extrinsic information $\lambda_{2}\left[b_{k}(n)\right]$ delivered by the channel decoder. This extrinsic information is the information about the code bit $b_{k}(n)$ gleaned from the prior information about the other code bits, $\left\{\lambda_{1}^{\mathrm{p}}\left[b_{k}(m)\right]\right\}_{m \neq n}$, based on the trellis constraint of the code. The SISO channel decoder also computes the a posteriori LLR of every information bit, which is used to make decision on the decoded bit at the last iteration. After interleaving, the extrinsic information delivered by the $K$ channel decoders $\left\{\lambda_{2}\left[b_{k}(i)\right]\right.$, $k=1, \ldots, K\}$ is then fed back to the SISO multiuser detector, as the prior information about the code bits of all users, in the next iteration. Note that at the first iteration, the extrinsic information $\left\{\lambda_{1}\left[b_{k}(i)\right]\right.$ and $\left\{\lambda_{2}\left[b_{k}(i)\right]\right.$ are statistically independent. But subsequently since they use the same information indirectly, they will become more and more correlated and finally the improvement through the iterations will diminish.

\section{Simulation Example}

We now illustrate the performance of the Turbo multiuser receiver in a multipath CDMA channel. We consider an asynchronous CDMA system with four users. The user spreading sequences are derived from Gold sequences of length seven. The number of paths for each user is three. The signature sequences, path delays and complex path gains for each user are randomly generated. All users employ the same rate $1 / 2$ constraint length 5 convolutional code. Each user uses a different random interleaver. The same set of interleavers is used for all simulations. The block size of the information bits for each user is 128 . In the simulation, the four user signals have equal power. The bit error rate (BER) curves of each user after the first 5 iterations are shown in Figure 5. The BER curve for the single-user channel is also plotted in the same figure as the dashed lines. It is seen that significant performance gain is achieved by the proposed iterative receiver structure compared with the non-iterative receiver structure (i.e., linear MMSE demodulator followed by soft channel decoder). Moreover, at high signal-to-noise ratio, the detrimental effects of the MAI and ISI in the channel can almost be completely eliminated and single-user performance can be approached.
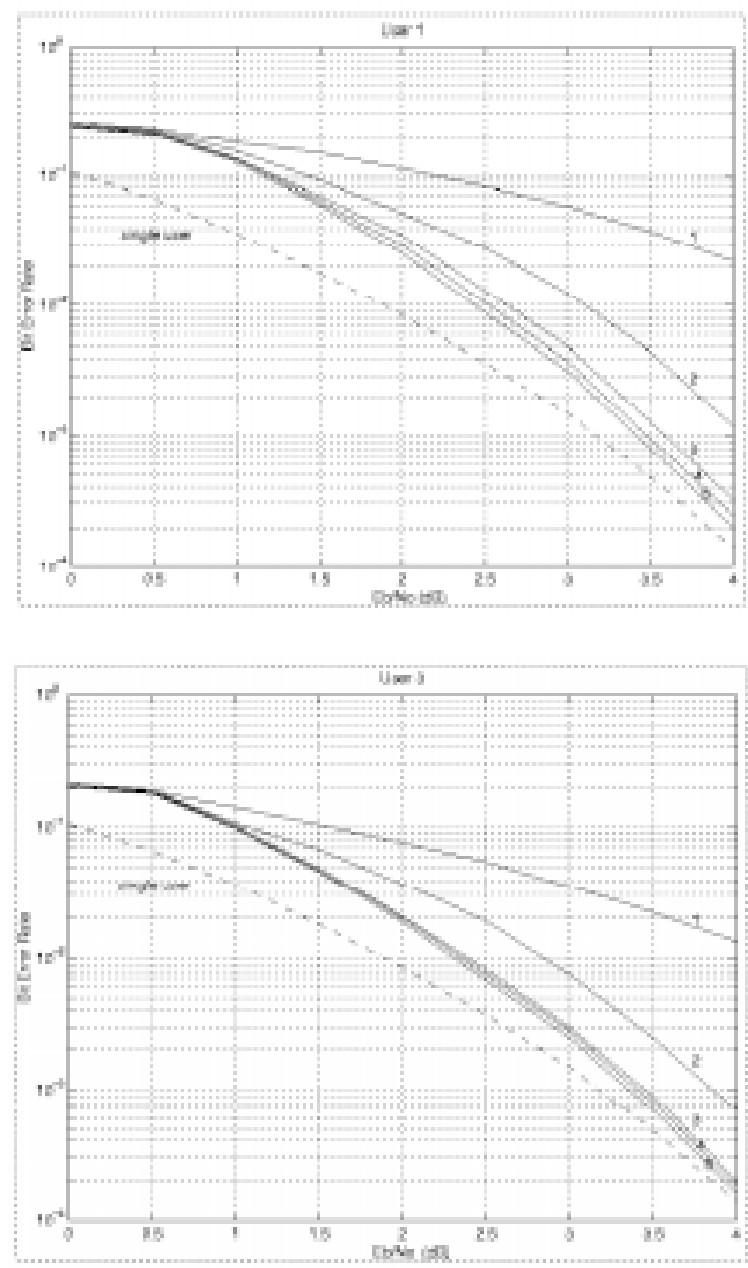

Figure 5: Performance of Turbo multiuser receiver in a 4user multipath channel. All users have equal power.

\section{Further Issues}

\section{Space-Time Processing}

The subspace blind multiuser detection and the Turbo multiuser detection techniques discussed in the preceding sections exploit the temporal structures of the multiple-access signals that are 


\section{Signal Processing}

either induced by signal multiplexing or by channel coding. Another very promising approach to interference suppression in wireless systems is through space-time processing using an antenna array at the receiver. In this approach, the signal structure induced by multiple receiving antennas, i.e., spatial signature, is exploited for interference rejection. Combined multiuser detection and array processing, so-called space-time multiuser detection, that exploits both the temporal and the spatial structures of the signals, as been addressed more recently [30].

In this case, it is assumed that at the receiver an antenna array of $Q$ elements is employed. Assuming that each transmitter is equipped with a single antenna, then the baseband multipath channel between the $k$-th user's transmitter and the base station receiver can be modeled as a single-input multiple-output channel with the following vector impulse response

$\underline{h_{k}}(t)=\sum_{l=1}^{L} \underline{a}_{k l} g_{k l} \delta\left(t-\tau_{k l}\right)$

where $L$ is the number of paths in each user's channel, $g_{k l}$ and $\tau_{\mathrm{kl}}$ are respectively the complex gain and delay of the $l$-th path of the $k$-th user's signal, and $\underline{a}_{k l}=\left[a_{k l, 1} \ldots a_{k l, Q}\right]^{\mathrm{T}}$ is the array response vector corresponding to the $l$-th path of the $k$-th user's signal. The total received signal at the receiver is then the superposition of the signals from the $K$ users plus the additive ambient noise, given by

$$
\begin{aligned}
& \underline{r}(t)=\sum_{k=1}^{K} x_{k}(t) * \underline{h}_{k}(t)+\underline{v}(t) \\
& =\sum_{i=0}^{M-1} \sum_{k=1}^{K} A_{k} b_{k}(i) \sum_{l=1}^{L} \underline{a}_{k l} g_{k l} s_{k}\left(t-i T-\tau_{k l}\right)+\underline{v}(t),
\end{aligned}
$$

where $*$ denotes convolution; $\underline{v}(t)=\left[v_{1}(t) \ldots v_{Q}(t)\right]^{\mathrm{T}}$ is a vector of independent zero-mean complex white Gaussian noise processes.

The multiuser space-time receiver structure is illustrated in Figure 6. The front-end of the receiver consists of a bank of matched-filters, followed by a bank of array combiners and then followed by a bank of multipath combiners, which produces the sufficient statistic. The maximum likelihood multiuser sequence detector or the linear multiuser detectors can be employed to demodulate the transmitted symbols of the users. It is demonstrated in [30] that significant performance gains can be obtained by combining space-time processing with multiuser detection. Moreover, it is straightforward to extend the subspace blind multiuser detection and the Turbo multiuser detection methods discussed earlier to address the multiple antenna scenario.

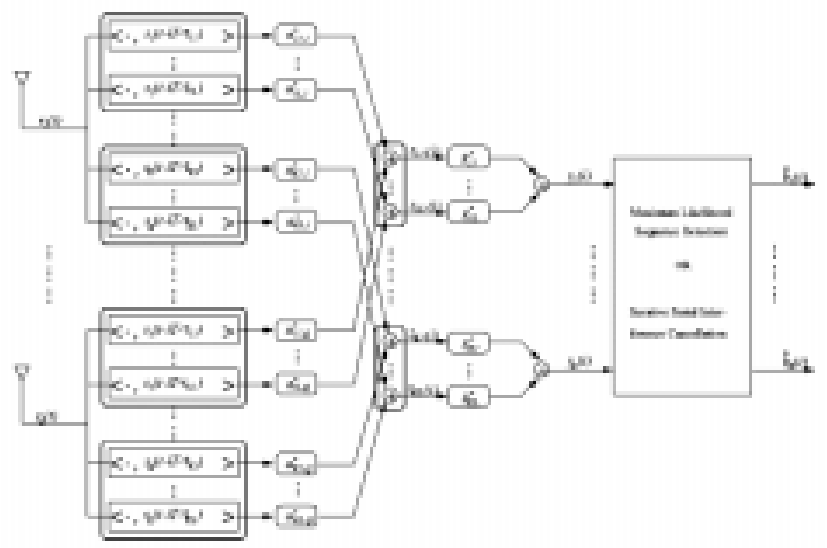

Figure 6: Space-time Multiuser receiver structure.

\section{Narrowband Interference Rejection}

One important type of interference arising from wideband wireless multimedia system is the narrowband interference (NBI). Such interference arises because of non-symmetric features of wireless systems, such as the desire to share bandwidth with other, dissimilar communication services (e.g., multi rate systems). Although spread spectrum CDMA communications are inherently resistant to the NBI, it has been demonstrated that the performance of wideband systems in the presence of NBI can be enhanced significantly through the use of active NBI suppression: Not only does active suppression improve error-rate performance, but it also leads to increased system capacity and improved acquisition capability.

Over the past two decades, a significant body of research has been concerned with the development of techniques for active NBI suppression. Most techniques seek to form a replica of the narrowband signals that can be subtracted from the received signal before data demodulation takes place. Early work in this area focused on techniques based on the linear signal processing regimes of adaptive linear transversal filtering, and Fourier-domain filtering [15,31]. Recently, model-based techniques that employ non-standard signal processing methods, including nonlinear filtering techniques $[21,23]$ and multiuser detection techniques $[16,20,22]$, have been used for NBI mitigation. More recently, the blind linear MMSE multiuser detection techniques described in Section 3 have been shown to work quite well against combined MAI and NBI of all types $[18,19]$.

\section{Impulsive Ambient Noise Rejection}

Another source of radio-frequency interference in a wireless multimedia network is the impulsive channel ambient noise. Most research on interference rejection in wireless systems has 
focused on situations in which the ambient noise has a Gaussian distribution. For many physical channels arising from wireless applications, however, the ambient noise is known through experimental measurements to be decidedly nonGaussian, due to the impulsive nature of the man-made electromagnetic interference and a great deal of natural noise as well. This is particularly true of urban and indoor radio channels $[6,7,12,13,14]$. It is widely known in the single-user context that non-Gaussian noise can be quite detrimental to the performance of conventional systems based on the Gaussian assumption, whereas it can actually be beneficial to performance if appropriately modeled and ameliorated [11]. Neither of these properties is surprising. The first is a result of the lack of robustness of linear and quadratic type signal processing procedures to many types of non-Gaussian statistical behavior [11]. The second is a manifestation of the well known leastfavorability of Gaussian channels.

In view of the lack of realism of a Gaussian model for ambient noise arising in many wireless channels, natural questions arise concerning the applicability, robustness and performance of interference suppression techniques for non-Gaussian multiple access channels [17]. Although performance indices such as mean-square-error (MSE) and signal-to-interference-plusnoise ratio (SINR) for linear multiuser detectors are not affected by the distribution of the noise (only the spectrum matters), the more crucial bit-error rate can depend heavily on the shape of the noise distribution. The results of an early study of error rates in non Gaussian DS-CDMA channels are found in $[1,2,3]$, in which the performance of the conventional and modified conventional detectors is shown to depend significantly on the shape of the ambient noise distribution. In particular, impulsive noise can severely degrade the error probability for a given level of ambient noise variance. In the context of multiple-access capability, this implies that fewer users can be supported with conventional detection in an impulsive channel than in a Gaussian channel. However,

Since non-Gaussian noise can, in fact, be beneficial to system performance if properly treated [11], the problem of joint mitigation of structured interference and non-Gaussian ambient noise has received considerable recent interests. An approach to this problem for NBI suppression in spread-spectrum systems is described in [8]. Some very recent results that describe nonlinear adaptive methods for suppressing MAI in the presence of impulsive noise in CDMA communication systems are found in $[26,29]$. These nonlinear techniques are based on the $M$-estimation method for robust regression, and they offer substantial performance gains over linear detection methods in impulsive ambient noise.

\section{Conclusions}

The projections of rapid escalating demand for advanced wireless services such as multimedia present major challenges in the field of telecommunications, and meeting these challenges will require sustained technical innovation on many fronts. Of paramount importance in addressing these challenges is the development of suitable adaptive systems to perform advanced signal processing functions for wireless multiple-access communications in non-stationary and interference-rich environments. This is the problem addressed in this paper. We have focused primarily on the problem of jointly suppressing multiple-access interference (MAI) and intersymbol interference (ISI), which are the limiting sources of interference for the high data-rate wireless multimedia applications. We have first presented a signal subspace approach to blind joint suppression of MAI and ISI.

We have then described a powerful iterative technique for joint interference suppression and decoding, so-called Turbo multiuser detection, that is especially useful for wireless multimedia packet communications. We have also discussed space-time processing methods that employ multiple antennas for interference rejection and signal enhancement. Finally, we have touched briefly on the problems of suppressing narrowband interference and impulsive ambient noise, two other sources of radio-frequency interference present in wireless multimedia networks. Although the signal processing methodologies presented in this paper are the results of very recent research work and more effort is needed in refining them before their deployment in actual wireless systems, these powerful techniques will have a far reaching impact on future wireless multimedia communication systems.

\section{Acknowledgements}

The author wishes to thank Miss Y. Amy Huang for typing the manuscript.

\section{References}

[1] B. Aazhang and H.V. Poor. Peformance of DS/SSMA communications in impulsive channels - Part I: Linear correlation receivers. IEEE Trans. Commun., COM-35(11):1179--1187, Nov. 1987.

[2] B. Aazhang and H.V. Poor. Peformance of DS/SSMA communications in impulsive channels - Part II: Hard-limiting correlation receivers. IEEE Trans. Commun., COM-36(1):88--96, Jan. 1988.

[3] B. Aazhang and H.V. Poor. An analysis of nonlinear directsequence correlators. IEEE Trans. Commun., COM-37(7):723-731, July 1989.

[4] C. Berrou and A. Glavieux. Near optimum error-correcting coding and decoding: Turbo codes. IEEE Trans. Commun., COM-44(10), Oct. 1996. 


\section{Signal Processing}

[5] C. Berrou, A. Glavieux, and P. Thitimajshima. Near Shannon limit error-correction coding and decoding: Turbo codes. In Proc. 1993 International Conference on Communications (ICC'93), pages 1064--1070, 1993.

[6] K.L. Blackard, T.S. Rappaport, and C.W. Bostian. Measurements and models of radio frequency impulsive noise for indoor wireless communications. IEEE J. Select. Areas Commun., 11(7):991-1001, Sep. 1993.

[7] T.K. Blankenship, D.M. Krizman, and T.S. Rappaport. Measurements and simulation of radio frequency impulsive noise in hospitals and clinics. In Proc. 1997 IEEE Vehicular Technology Conference (VTC'97), pages 1942--1946, 1997.

[8] L.M. Garth and H.V. Poor. Narrowband interference suppression techniques in impulsive channels. IEEE Trans. Aerosp. Electron. Syst., 28(1):15--34, Jan. 1992.

[9] J.Hagenauer. The Turbo principle: Tutorial introduction and state of the art. In Proc. International Symposium on Turbo Codes and Related Topics, pages 1--11, Brest, France, Sep. 1997.

[10] A.Host-Madsen and X. Wang. Group-blind multiuser detection for uplink CDMA. In Proc. 1999 IEEE VTC, Houston, TX, May 1999.

[11] S.A. Kassam and H.V. Poor. Robust techniques for signal processing: A survey. Proc. IEEE, 73(3):433--481, Mar. 1985.

[12] D.Middleton. Man-made noise in urban environments and transportation systems: models and measurement. IEEE Trans. Commun., COM-21(11):1232--1241, Nov. 1973.

[13] D.Middleton. Statistical-physical models of electromagnetic interference. IEEE Trans. Electromag. Compat., EMC-19:106--127, 1977.

[14] D.Middleton and A.D. Spaulding. Elements of weak signal detection in non-Gaussian noise. In H. $\sim$ V. Poor and J. B. Thomas, editors, Advances in Statistical Signal Processing Vol. 2: Signal Detection. JAI Press, Greenwich, CT, 1993.

[15] L.B. Milstein. Interference rejection techniques in spread spectrum communications. Proc. IEEE, 76(6):657--671, June 1988.

[16] H.V. Poor and L. A. Rusch. Narrowband interference suppression in spread spectrum CDMA. IEEE Personal Communications Magazine, 1(3):14--27, Aug. 1994.

[17] H.V. Poor. Non-Gaussian signal processing problems in multipleaccess communications. In Proc. 1996 USC/CRASP Workshop on Non-Gaussian Signal Processing, Ft. George Meade, MD, May 1996.

[18] H.V. Poor and X.Wang. Code-aided interference suppression in DS/CDMA communications - Part I: Interference suppression ca- pability. IEEE Trans. Commun., COM-45(9):1101--1111, Sep. 1997.

[19] H.V. Poor and X. Wang. Code-aided interference suppression in DS/CDMA communications - Part II: Parallel blind adaptive implementations. IEEE Trans. Commun., COM-45(9):1112--1122, Sep. 1997.

[20] H.V. Poor and X. Wang. Blind adaptive suppression of narrowband digital interferers from spread-spectrum signals. Wireless Personal Communications , 6(1-2):69--96, Jan. 1998.

[21] L.A. Rusch and H.V. Poor. Narrowband interference suppression in CDMA spread spectrum communications. IEEE Trans. Commun., COM-42(2/3/4):1969--1979, Feb./Mar./Apr. 1994.

[22] L.A. Rusch and H.V. Poor. Multiuser detection techniques for narrowband interference suppression in spread spectrum communications. IEEE Trans. Commun., COM-43(2/3/4):1725--1737, Feb./Mar./Apr. 1995.

[23] R.Vijayan and H.V. Poor. Nonlinear techniques for interference suppression in spread spectrum systems. IEEE Trans. Commun., COM-38(7):1060--1065, July 1990.

[24] X.Wang and H.V. Poor. Blind equalization and multiuser detection for CDMA communications in dispersive channels. IEEE Trans. Commun., COM-46(1):91--103, Jan. 1998.

[25] X.Wang and H.V. Poor. Blind multiuser detection: A subspace approach. IEEE Trans. Inform. Theory, 44(2):677--691, Mar. 1998.

[26] X.Wang and H.V. Poor. Robust adaptive array for wireless communications. IEEE J. Select. Areas Commun., 16(8):1352--1367, Oct. 1998.

[27] X.Wang and H.V. Poor. Blind joint equalization and multiuser detection for DS-CDMA in unknown correlated noise. IEEE Trans. Circuits and Systems - II: Analog and Digital Signal Processing, 46(7), July. 1999.

[28] X. Wang and H.V. Poor. Iterative (Turbo) soft interference cancellation and decoding for coded CDMA. IEEE Trans. Commun., 47(7), July. 1999.

[29] X.Wang and H.V. Poor. Robust multiuser detection in nonGaussian channels. IEEE Trans. Sig. Proc., 47(2):289--305, Feb. 1999.

[30] X.Wang and H.V. Poor. Space-time multiuser detection in multipath CDMA\}channels. IEEE Trans. Sig. Proc., 47(9), Sept. 1999.

[31] P. Wei, J.R. Ziedler, and W.H. Ku. Adaptive interference suppression for \{CDMA $\}$ overlay systems. IEEE J. Select. Areas Commun., 12(9):1510--1523, Dec. 1994. 